\title{
Mesoporous Bragg reflectors - block-copolymer self-assembly leads to building blocks with well defined continuous pores and high control over optical properties
}

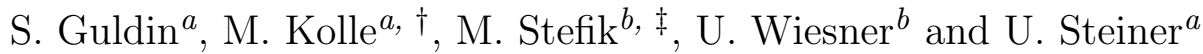 \\ ${ }^{a}$ Department of Physics, University of Cambridge, JJ Thomson Ave, Cambridge CB30HE, UK; \\ ${ }^{b}$ Department of Materials Science \& Engineering, Cornell University, Ithaca, NY 14853, USA; \\ $\ddagger$ Now at: Harvard School of Engineering and Applied Sciences, Cambridge, MA 02138, USA; \\ $\ddagger$ Now at: Institute of Physical Chemistry, École Polytechnique Fédérale de Lausanne, CH
}

\begin{abstract}
Mesoporous distributed Bragg reflectors (MDBRs) exhibit porosity on the sub-optical length scale. This makes them ideally suited as sensing platforms in biology and chemistry as well as for light management in optoelectronic devices. Here we present a new fast forward route for the fabrication of MDBRs which relies on the self-assembling properties of the block copolymer poly(isoprene-block-ethylene oxide) (PI- $b$-PEO) in combination with sol-gel chemistry. The interplay between structure directing organic host and co-assembled inorganic guest allows the fine tuning of refractive index in the outcome material. The refractive index difference between the high and low porosity layer can be as high as 0.4, with the optical interfaces being well defined. Following a 30 min annealing protocol after each layer deposition enables the fast and reliable stacking of MDBRs which exhibit a continuous $\mathrm{TiO}_{2}$ network with large accessible pores and high optical quality.
\end{abstract}

Keywords: polymer, self-assembly, porosity, Bragg reflector, photonic crystal, $\mathrm{TiO}_{2}$

\section{INTRODUCTION}

A distributed Bragg reflector (DBR) is defined as a one-dimensional (1D) periodic dielectric lattice of alternating high and low refractive index materials. Reflection of light at each of the layer interfaces leads to multiple interference and thus, a characteristic reflection and transmission behavior. The structural color of the multilayer stack can be finely tuned by varying the refractive index, the thickness, and the number of the alternating layers. ${ }^{1}$ DBRs are of technological interest because of their spectrally selective wave guiding properties. Well established applications include Fabry-Pérot interferometers, optical resonators for distributed feedback lasers, and optical cavities for controlling the spontaneous emission rates and spectra from emitting media. ${ }^{2,3}$ Simple configurations are realized by sequential coating of thin solid films of alternating refractive index. Organic DBRs that are stretch-tunable throughout the visible spectrum have also been demonstrated. ${ }^{4}$

The development of mesoporous DBRs (MDBRs) has in recent years evolved into an active field of research. Mesoporous, as the exhibit accessible pores on the sub-optical length scale, which opens up a number of new and unique applications. Their ability to detect changes in the ambient environment makes them ideally suited as sensing materials. Adsorption and desorption of gas phase molecules in the pores leads to reversible changes in the refractive index of the building blocks, which changes its photonic properties. ${ }^{5,6}$ Functionalized to bind specific molecules MDBRs have been successfully used as biosensors. ${ }^{7}$ Bonifacio et al. introduced the concept of an array of MDBRs with different functionalizations to detect and discriminate different vapor species such as small molecules and bacteria volatiles. ${ }^{8}$ The coupling of MDBRs to surface layers ${ }^{9}$ and resonance cavities ${ }^{10}$ is particularly attractive as it allows sensing with greater accuracy. Concepts on how to profit from the optical properties of MDBRs for optoelectronic devices are plentiful. Distributed feedback lasing has been reported

Further author information:

S.G.: E-mail: sg506@cam.ac.uk, Telephone: +44 1223337285

U.S.: E-mail: u.steiner@phy.cam.ac.uk , Telephone: +44-1223-337390

Active Photonic Materials IV, edited by Ganapathi S. Subramania, Stavroula Foteinopoulou,

Proc. of SPIE Vol. 8095, 80951G · @ 2011 SPIE · CCC code: 0277-786X/11/\$18 · doi: 10.1117/12.893818

Proc. of SPIE Vol. $809580951 \mathrm{G}-1$ 
after infiltrating the porous structure with light emitting polymers. ${ }^{11}$ In the field of dye-sensitized solar cells the localization of light of a specific part of the spectrum has led to efficiency enhancements due to the increase in absorption probability. ${ }^{12}$

The material routes towards MDBRs are numerous. Initially, the 1D refractive index lattice was realized in porous silicon where etching conditions were periodically changed to achieve high and low porosity layers in alternating order. ${ }^{13}$ In the mid 2000s Ozin and coworkers introduced the concept of solution-processed MDBRs, by the alternating spin coating of surfactant $\mathrm{P} 123$-assembled mesoporous $\mathrm{TiO}_{2}$ and $\mathrm{SiO}_{2}$ layers. ${ }^{5}$ While the morphology within the network showed good structural order, processing was extremely time consuming with fabrication times up to several days for each individual layer of the stack. ${ }^{14}$ A less complex route to form porous $\mathrm{TiO}_{2}$ and $\mathrm{SiO}_{2}$ networks is the random packing of nanoparticles. This was introduced by Cohen et al. ${ }^{15}$ and further simplified by Miguez et al. ${ }^{16}$ The optical properties of nanoparticle-based MDBRs can be tuned by changing the thickness and the materials base of the building blocks (e.g. $\mathrm{TiO}_{2}$ and $\mathrm{SiO}_{2}$ ), but the control over porosity proves difficult. The random-close packing of the particles results in a rather polydisperse pore size distribution and allows only limited control over the porosity by changing the nanoparticle size. ${ }^{17}$ The refractive index contrast is therefore typically achieved by stacking $\mathrm{TiO}_{2}$ and $\mathrm{SiO}_{2}$ layers of similar porosity, which leads to insulating MDBRs - particulary disadvantageous in optoelectronic devices.

Herein, we present an alternative concept for the fabrication of MDBRs based on single material but alternating porosity. The refractive index of the building blocks can be finely tuned by the interplay between the self-assembly of the block copolymer host PI- $b$-PEO and the co-assembled $\mathrm{TiO}_{2}$, which is derived by hydrolytic sol-gel chemistry. Alternating deposition of high and low refractive index layers enables the stacking of a MDBR with unique properties. These include the fast and reliable assembly of a continuous network, fine control over the pore size distribution, well defined optical interfaces, and thus components of high optical quality.

\section{EXPERIMENTAL}

\subsection{Fabrication of MDBR stack}

Two stock solution with a differing ratio of $\mathrm{TiO}_{2}$ sol to PI- $b$-PEO block copolymer were prepared in the following way. First, a titanium containing sol was obtained by the rapid addition of $0.69 \mathrm{ml} \mathrm{HCl} \mathrm{(37 \% )} \mathrm{to} 1 \mathrm{ml}$ titanium ethoxide (purum) under vigorous stirring. In parallel, two polymer solutions were prepared by dissolving $0.1 \mathrm{~g}$ of PI- $b$-PEO (molecular weight $M_{\mathrm{n}}=34.4 \mathrm{~kg} \mathrm{~mol}^{-1}, 28 \mathrm{wt} \% \mathrm{PEO}$ ) in $7 \mathrm{ml}$ tetrahydrofuran (THF). After stirring for $90 \mathrm{~min}$, the sol was added to the polymer solution. The weight ratio of 3:1 ("titania 3:1") and 1:2 ("titania 1:2") between the resulting $\mathrm{TiO}_{2}$ and the initially used polymer were chosen for the high and low refractive index layers, respectively.

For "titania 3:1", $1.31 \mathrm{ml}$ sol was added, followed by the evaporation of volatile components at $50{ }^{\circ} \mathrm{C}$ in a Petri dish. Once dry, the hybrid material was quickly redissolved in a in a solvent mixture of $49 \mathrm{vol} \%$ toluene and $51 \mathrm{vol} \%$ 1-butanol. The "titania 1:2" was prepared similarly, but in this case $0.22 \mathrm{ml}$ sol was added to the polymer base solution and the solvent mixture for redissolving the dried hybrid material consisted of an azeotrope mixture of $73 \mathrm{vol} \%$ toluene and $27 \mathrm{vol} \%$ 1-butanol. Subsequently the concentrations of the hybrid solutions were adjusted to match the film thickness requirements. Films were then deposited by spin coating (2000 rpm, $20 \mathrm{~s})$ onto previously cleaned microscope slides. Subsequent to each film layer deposition, an annealing cycle of $10 \mathrm{~min}$ at $100^{\circ} \mathrm{C}, 200^{\circ} \mathrm{C}$ and $300^{\circ} \mathrm{C}$ was applied. Layers from "titania $3: 1$ " and "titania $1: 2$ " were deposited in alternating fashion to build-up the MDBR stack. Finally the MDBR was calcined at $500{ }^{\circ} \mathrm{C}\left(3\right.$ hours, heat ramp $\left.5{ }^{\circ} \mathrm{C} \mathrm{min}{ }^{-1}\right)$ for the removal of the organic material and crystallization of the $\mathrm{TiO}_{2}$.

\subsection{Material characterization}

Imaging was carried out by scanning and transmission electron microscopy (SEM, TEM). For SEM, a Leo Ultra 55 was used with a field emission source of $3 \mathrm{kV}$ acceleration voltage. TEM was performed on a FEI Philips Tecnai 20 system with $200 \mathrm{kV}$ acceleration voltage. The cross-section was obtained by focused ion beam milling. Spectroscopic characterization of the reflection properties was carried out on a Olympus BX51 microscope $(5 \times$ magnification). A Ocean QE 65000 spectrometer served for detection of the signal. Imaging ellipsometry was accomplished on a Nanofilm ep3se device. The instruments software was used to analyze the data. For modeling of

Proc. of SPIE Vol. 8095 80951G-2 
(a)

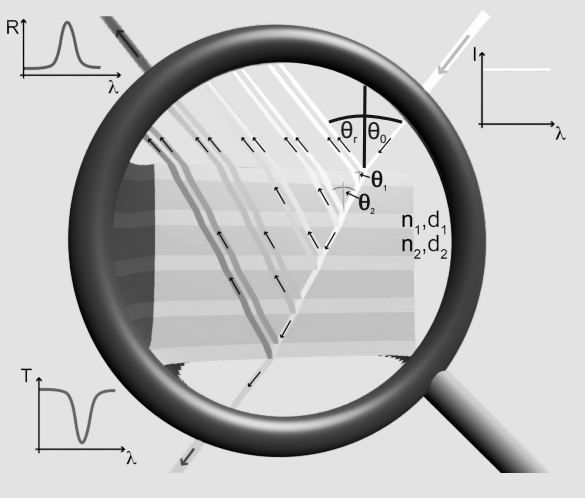

(c)

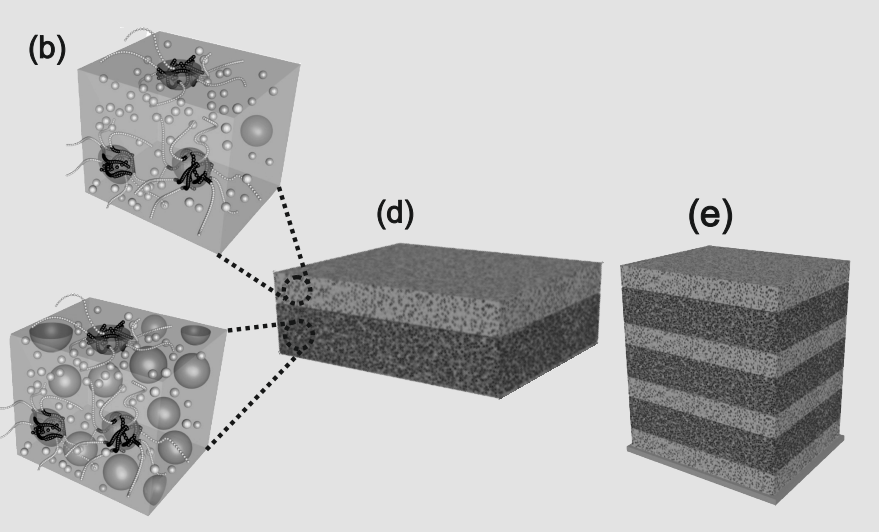

Figure 1. Mesoporous Bragg reflectors (MDBRs) - functioning and fabrication. (a) MDBRs consist of a periodic lattice of alternating high and low refractive index, while exhibiting porosity on the sub-optical length scale. Multiple interference at the interfaces of the dielectric layers leads to characteristic reflection and transmission properties, which can be finely tuned by adjusting the refractive index and layer thickness of the building blocks. (b)-(e) Schematic of the fabrication method. Two solutions of $\mathrm{TiO}_{2}$ sol and PI- $b$-PEO block copolymer in different weight ratios (3:1 \& 1:2) serve as fabrication base. Spin-coating of the "titania 3:1" solution leads to a $\mathrm{TiO}_{2}$ - rich hybrid network, shown in (b), while reducing the ratio in "titania 1:2" results in a mainly organic composition (i.e. more porous after the removal of the organics), illustrated in (c). A 30 min temperature protocol after each film deposition anneals the film and makes it resistant to the consecutive spin-coating of additional layers (d). For the build-up the stack, solutions of the different weight ratios are spin-coated alternately, each time followed by a $30 \mathrm{~min}$ annealing step (e). Removal of the organic material in a final calcination step at $500{ }^{\circ} \mathrm{C}$ results in a crystalline $\mathrm{MDBR}$ of $\mathrm{TiO}_{2}$, whose photonic properties originate from the alternating sequence of high and low porosity layers.

the MDBR stacks, a Matlab algorithm was developed based on Rouard's technique. ${ }^{1}$ The data was subsequently verified using a transfer matrix algorithm coded in $\mathrm{C}^{++}$.

\section{RESULTS AND DISCUSSION}

The physical origin behind the functioning of DBRs is illustrated in Figure 1a. Due to the refractive index contrast between the dielectric layers, incoming light is partially reflected at each of the layer interfaces. These $(N+1)$ reflections (with $N$ being the number of layers in the stack) lead to multiple interference and therefore a characteristic reflection and transmission behavior of the incoming light. The Bragg-Snell law describes the position of the central DBR stop-band at normal incidence for non-absorbing materials ${ }^{18}$ :

$$
m \lambda_{\max }=2\left(n_{\mathrm{l}} h_{\mathrm{l}}+n_{\mathrm{h}} h_{\mathrm{h}}\right)
$$

with $m$ being the diffraction order, and $n_{1, \mathrm{~h}}$ and $h_{1, \mathrm{~h}}$ being the refractive index and layer thickness of the high and low refractive index material, respectively. The bandwidth $\Delta \lambda_{\max }$ (full width at half maximum) of the central Bragg peak is defined as

$$
\Delta \lambda_{\max }=\frac{4 \lambda_{\max }}{\pi} \arcsin \left(\frac{n_{\mathrm{h}}-n_{\mathrm{l}}}{n_{\mathrm{h}}+n_{\mathrm{l}}}\right)
$$

The reflectivity can be described with

$$
R=\left(\frac{n_{\text {surrounding }}-n_{\text {substrate }}\left(n_{\mathrm{l}} / n_{\mathrm{h}}\right)^{2 N}}{n_{\text {surrounding }}-n_{\text {substrate }}\left(n_{\mathrm{l}} / n_{\mathrm{h}}\right)^{2 N}}\right)^{2}
$$

The parameters for tuning the structural coloration of the stack are therefore the absolute values of $n_{\mathrm{h}}$ and $n_{\mathrm{l}}$, their difference $\Delta n$, the thickness of the building blocks, as well as the number of the alternating layers.

The block copolymer PI- $b$-PEO plays a key role in our fabrication route, illustrated in Figure 1b-e. Due to the incompatibility of its covalently linked blocks, the macromolecule microphase separates into ordered domains 

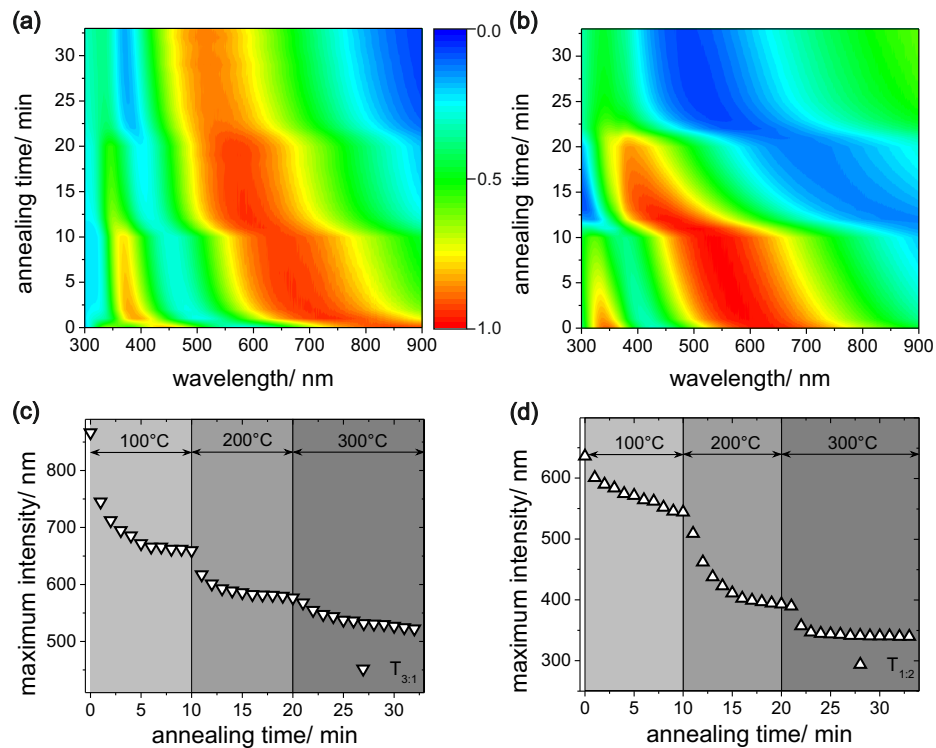

Figure 2. Annealing of block copolymer assembled hybrid films. Films suffer from significant shrinkage during the several processing steps. In its final calcined state, "titania 3:1" films have shrunk to $42 \%$, "titania 1:2" films to $33 \%$ compared to its initial thickness after spin-coating. ${ }^{20}$ In order to prevent cracking and delamination, time- and temperature-dependent shrinkage was studied in detail. Results led to the establishment of a 30 min annealing protocol with temperature steps at $100^{\circ} \mathrm{C}, 200^{\circ} \mathrm{C}$, and $300^{\circ} \mathrm{C}$, for $10 \mathrm{~min}$ each. Reflection spectroscopy of the hybrid film during the annealing process on a hotplate allows in-situ monitoring of the shrinkage process. The reflection intensity for a "titania 3:1" film is shown in (a), reflection of a "titania 1:2" represented in (b). The shrinkage of the films corresponds to a shift of the reflectance maxima and minima to smaller wavelengths. The three steps at $100{ }^{\circ} \mathrm{C}(0-10 \mathrm{~min}), 200^{\circ} \mathrm{C}(10-20 \mathrm{~min})$, and $300^{\circ} \mathrm{C}(>20 \mathrm{~min})$, can be clearly identified in the contour plots. For clarity, the position of the reflectance maximum is plotted below for "titania 3:1" (c), and "titania 1:2" (d), respectively. The change in reflectance suggests that most of the shrinkage at each temperature occurs within the 10 min interval.

on the 5 to $50 \mathrm{~nm}$ length-scale. In the herein presented route the polymer acts as a host for the inorganic material. The nanoparticle sol selectively swells the PEO block, and therefore takes part in the self-assembly process. Upon calcination in a high temperature step, the mesoporous inorganic material then resembles the polymer microphase morphology imposed on it. See the pioneering work of Templin et al. for further details. ${ }^{19}$ Due to this interplay between the block copolymer and the nanoparticle sol, the refractive index of the resulting inorganic material can be finely tuned by the mixing ratio of the components in the initial solution. In our case, we prepared solutions with mixing ratios of $3: 1$ ("titania 3:1") and 1:2 ("titania 1:2") between the $\mathrm{TiO}_{2}$ and the block copolymer. Alternating deposition of high and low refractive index layers then enables the stacking of a MDBR before the organic material is removed in a final calcination step at $500^{\circ} \mathrm{C}$.

A major challenge for the fabrication of block-copolymer derived mesoporous films is the considerable shrinkage, which occurs during the several processing steps. The volume contraction due to the condensation of the inorganic network, removal of the polymer and crystallization of the $\mathrm{TiO}_{2}$ can lead to crack formation and delamination for films beyond several hundred nm. ${ }^{21}$ Our aim was to establish a processing protocol, which accommodates the shrinkage step-by-step, but keeps the necessary fabrication time to a minimum. The use of optical means enabled us to not only study the temperature dependence but also resolve the shrinkage kinetics in detail. ${ }^{20}$ Therefor, we collected the reflection spectra of the films during the annealing on a hotplate.The development of the optical film thickness $(n \cdot h)$ can then be resolved by the position of the interference fringes. Figure $2 \mathrm{a}$ and $\mathrm{b}$ show the time resolved development of the reflection spectra ( 1 min intervals, normalized to that of a blank silicon wafer) in a contour plot for "titania 3:1" and "titania 1:2", respectively. Films were subject to an annealing protocol of $10 \mathrm{~min}$ at each temperature of $100{ }^{\circ} \mathrm{C}, 200^{\circ} \mathrm{C}$ and $300^{\circ} \mathrm{C}$. The choice of these three temperature steps was based on previous results for thermogravimetric analysis and ex-situ thickness tracking. ${ }^{20}$ 

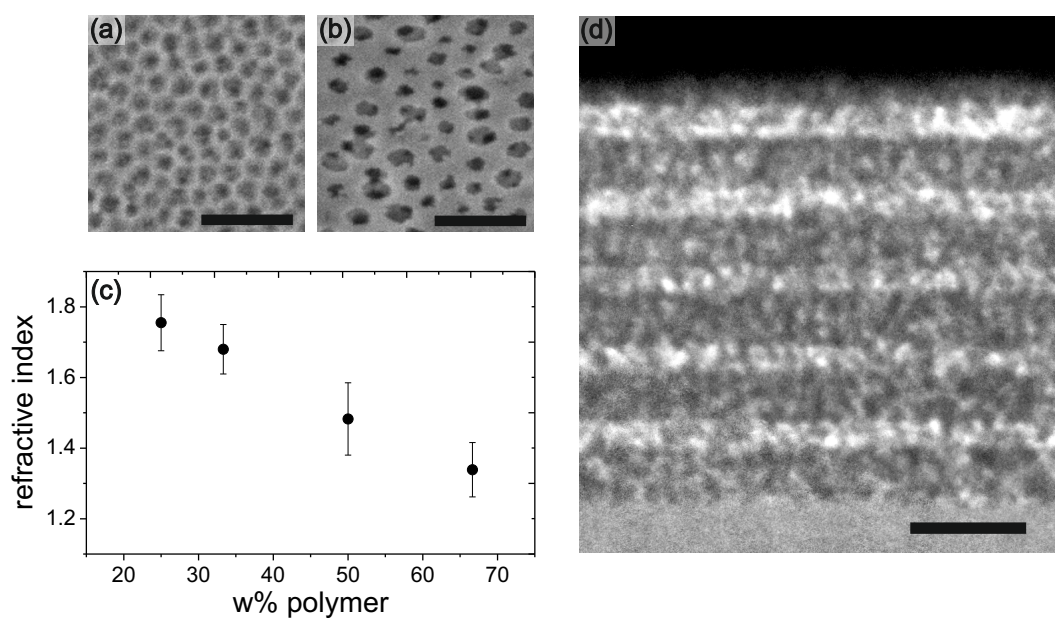

Figure 3. Electron microscopy of the building blocks after calcination at $500{ }^{\circ} \mathrm{C}$. (a) Top-view of a highly porous "titania 1:2" layer with a scanning electron microscope (SEM). (b) SEM top-view of a more dense "titania 3:1" layer. The variation of the mixing ratio between sol and structure-directing block copolymer allows the fine tuning of porosity and therefore refractive index in the resulting inorganic layers, shown in (c). The optical properties of the building blocks were characterized by imaging ellipsometry. (d) Transmission electron micrograph of a cross-section of a 9-layer MDBR. The contrast between darker "titania 3:1" and brighter "titania 1:2" layers is due to the differing electron density of the porous layers. Scale bars are $200 \mathrm{~nm}$ for (a), (b) and (d).

The three individual steps can be clearly seen in the reflection characteristics, with the reflectance maxima and minima moving to smaller wavelengths. For clarity, the position of the reflectance maximum is shown as a function of the annealing time in Figure 2c and d for "titania 3:1" and "titania 1:2", respectively. Shrinkage of the predominantly inorganic film "titania $3: 1$ " is significantly faster than for mostly organic "titania 1:2". The development of the peak suggests that in both cases most of the shrinkage at each temperature occurs within the 10 min interval. Please see ref $^{22}$ for further details on shrinkage in block copolymer derived mesoporous films.

Stacking is therefore realized by alternating between film deposition and film annealing. In a final high temperature step at $500{ }^{\circ} \mathrm{C}$, the stack is calcined, which removes the organic material, reveals the pores, and crystallizes the $\mathrm{TiO}_{2}$ network. Scanning electron micrographs of calcined "titania 1:2" and "titania 3:1" films are shown in Figure 3a and b, respectively. The differing porosity of films stemming from different mixing ratios is clearly discernible. "Titania 1:2" exhibits a very open pore structure with thin walls being surrounded by a large volume fraction of pores. In contrary, "titania 3:1" shows a more dense structure, with similar pore sizes but a much smaller pore density. The effect of the polymer $\mathrm{w} \%$ on the refractive index of the resulting mesoporous film is shown in Figure 3c. The results are in line with expected values derived from the Bruggemann effective medium approximation. ${ }^{23}$ In contrast to earlier work, ${ }^{24}$ no solid $\mathrm{TiO}_{2}$ overlayer was observed in any thin film. These results imply that in the given $\mathrm{w} \%$ range the organic structure directing agent is able to incorporate the full amount of inorganic material into the PEO block, enabling reliable phase separation and refractive index tuning without segregation of inorganic material. The considerable variation in $n$ from batch to batch may be due to the uncertainties involved in handling the masses of inorganics (in the sol) and organics (in solution). In contrast, the variation of $n$ from film to film within the same batch is negligible. The refractive index difference $(\Delta n>0.4)$ is only generated by the large variation in porosity of the resulting material, which is significantly higher to previously reported results. ${ }^{17}$ Figure 3 d shows a cross-section of the inorganic stack, prepared by focussed ion bean milling and imaged by transmission electron microscopy. The regularity of the stack is demonstrated by the exact lattice of high and low electron density layers, stemming from the alternating building blocks of low ("titania 3:1") and high porosity ("titania 1:2"), respectively.

Figure 4 shows the optical performance of the resulting MDBRs. In Figure 4a, the reflectance of different 9 layer MDBRs is compared. In all samples, the five layers "titania 3:1" were kept constant at $\approx 75 \mathrm{~nm}$, while the thickness of the "titania 1:2" layers was varied between $78 \mathrm{~nm}$ and $292 \mathrm{~nm}$ to tune the characteristic reflectance 

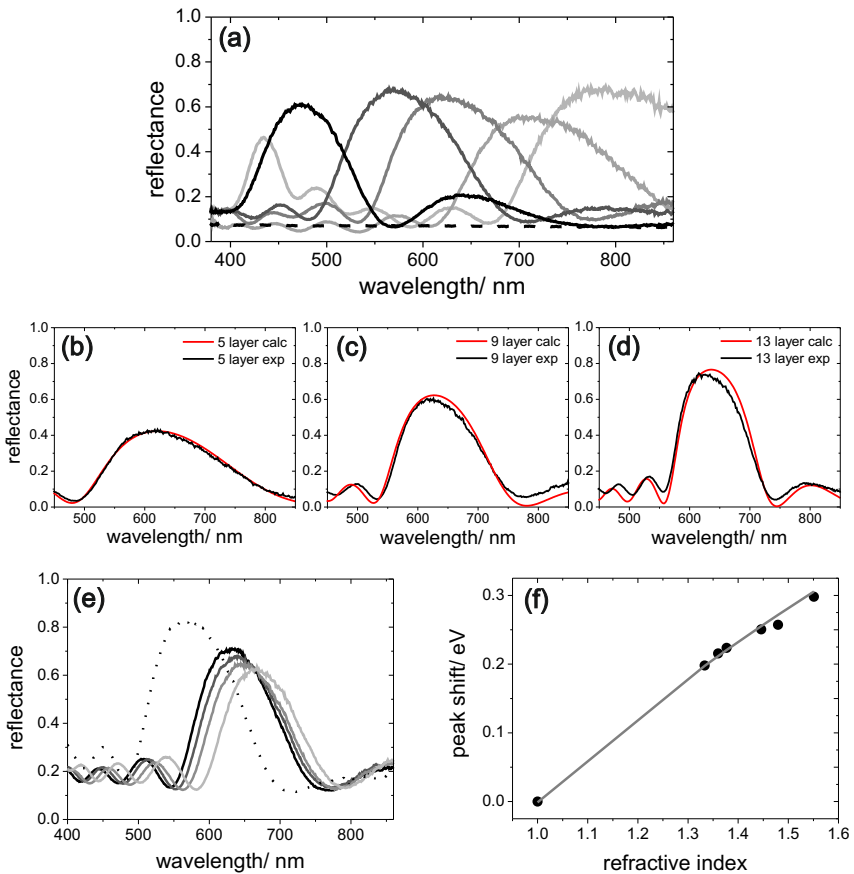

Figure 4. Optical properties of the MDBRs. (a) Reflectance of MDBRs, where the five layers "titania 3:1" were kept constant, while the four layers "titania 1:2" were varied between $78 \mathrm{~nm}$ and $292 \mathrm{~nm}$ to tune the characteristic reflectance throughout the visible spectrum. (b-d) Comparison of experimental reflectance with calculations of an ideally regular stack for 5 (b), 9 (c), and 13 (d) layers, respectively. The optical properties of the individual building blocks were determined by ellipsometry. Modeling was done using a Matlab algorithm based on Rouard's technique. ${ }^{1}$ (e) Reflectance of MDBR stack in air (dotted line) and after infiltration with $\mathrm{H}_{2} \mathrm{O}$, 2-propanol, chloroform, and 1,2-dichlorobenzene, plotted from dark grey to light grey with increasing refractive index. (f) Corresponding shift of the Bragg peak position as a function of the refractive index for 6 different infiltrated liquids. The grey line corresponds to the theoretical shift of the Bragg peak calculated by the symmetric Bruggeman effective medium approximation. These results confirm pore volumes of 0.265 and 0.540 for "titania $3: 1 "$ and "titania $1: 2 "$, respectively.

peak from $\approx 475 \mathrm{~nm}$ to $\approx 800 \mathrm{~nm}$, i.e. throughout the visible spectrum. In order to evaluate the regularity of the multilayer stack, the experimental results were compared to theoretical calculations. For this purpose, the $n$ and $h$ of the individual layers were determined by imaging ellipsometry $\left(n_{3: 1} \approx 1.70 \pm 0.02, h_{3: 1} \approx 76 \pm 2 \mathrm{~nm}\right.$ and $n_{1: 2} \approx 1.40 \pm 0.02, h_{1: 2} \approx 132 \pm 2 \mathrm{~nm}$ ). modeling was then carried out using a home-built Matlab algorithm, based on Rouard's technique. ${ }^{1}$ The modeled optical response of an ideal lattice is compared in Figure $4 \mathrm{~b}, \mathrm{c}$, and $\mathrm{d}$ for 5, 9, and 13 layers, respectively. These results demonstrate the high optical quality of the fabrication route, which is a direct consequence of the well defined optical interfaces, as well as the highly reproducible thickness and refractive index of the layers. The interconnectivity of the pores throughout the multiple layers is evidenced in Figure 4e. The reflection spectra of a 9 layer MDBR in air is here compared to its photonic properties after infiltration of the porous network with $\mathrm{H}_{2} \mathrm{O}, 2$-propanol, chloroform, and 1,2-dichlorobenzene. The position of the corresponding first order Bragg peak is plotted in $4 \mathrm{f}$ as a function of the refractive index of 6 different solvents. For full infiltration of the porous network, the peak shift should follow the development of $n$ according to the effective refractive index approximation (grey line). ${ }^{23}$ The good agreement is further supported by results from imaging ellipsometry, revealing accessible pore volumes of 0.265 and 0.540 for "titania 3:1" and "titania 1:2", respectively.

The herein presented materials route offers several advantages over the state-of-the-art. In contrast to nanoparticle-based approaches, the porosity can be finely tuned over a wide parameter space. Furthermore, we have shown in earlier work that the pore size in the resulting films can be controlled, as it is determined by the molecular weight of the PI-block of the polymer. ${ }^{25}$ This route therefore decouples two important parameters 
in MDBR stack design - porosity and pore size, and allows both properties to be tuned in a wide parameters space. Compared to random networks of nanoparticles, the pore size distribution is narrow and controllable. ${ }^{26}$ The use of block copolymer derived $\mathrm{TiO}_{2}$ has furthermore superior properties when used as electrode material for solid state dye-sensitized solar cells. We have previously shown improved conductivity compared to nanoparticle films due to the spatial confinement of $\mathrm{TiO}_{2}$ domains, ${ }^{27}$ as well as the manipulation of sub-bandgap states, ${ }^{28}$ both aspects being particular important for this device type. Previous approaches to polymer-directed MDBR fabrication were limited by the extremely long fabrication times and the variation in porosity and pore size for one specific material. ${ }^{5,14}$ The present route is fundamentally different due to the properties of the structure directing agent PI- $b$-PEO. The high Flory-Huggins interaction parameter of the isoprene and ethylene oxide blocks, their low glass-transition temperatures, and the high molecular weight are the main reasons why microphase separation occurs orders of magnitude faster than in the commercially available Pluronic P123 (poly(ethylene oxide)- $b$-(propylene oxide)- $b$-(ethylene oxide)). On the other hand it allows a large variation in the mixing ratio between the organic host and the inorganic guest and therefore fine control over the refractive index in the

mesoporous films. Moreover, charge carrier mobilities are superior as structural stability and fidelity during calcination can be maintained up to $700^{\circ} \mathrm{C} .{ }^{27}$

\section{CONCLUSIONS}

In conclusion, we present a new materials route to mesoporous Bragg reflectors. The self-assembly process imposed on the $\mathrm{TiO}_{2}$ sol and driven by the block copolymer host PI- $b$-PEO enables fine control over the porosity and refractive index in the resulting mesoporous layers. A 30 min annealing protocol per layer ensures sharp optical interfaces and supports the build-up of a crack-free stack. The resulting MDBRs exhibit a continuous network of pores with well defined dimensions, allowing full infiltration of the film with other functional materials. There is now an abundant range of sol-gel chemistry-based approaches which are compatible with block-copolymer assembly. ${ }^{29,30}$ It is therefore possible to extend the presented $\mathrm{TiO}_{2}$-based MDBRs to a wide range of ceramics and metal oxides. With the fine control over pore size, porosity and material composition, this approach presents new opportunities for MDBR manufacture and integration into functional devices.

\section{ACKNOWLEDGMENTS}

This publication is based on work supported in part by Award No. KUS-C1-018-02, made by King Abdullah University of Science and Technology (KAUST), the EPSRC (EP/F056702/1 and EP/F065884/1), the Department of Energy (DE-FG02 87ER45298) through the Cornell Fuel Cell Institute (CFCI) and the National Science Foundation (DMR-0605856). M.S. was supported by the Cornell Fuel Cell Institute and the Energy Materials Center at Cornell (EMC2), an Energy Frontier Research Center funded by the U.S. Department of Energy, Office of Science, Office of Basic Energy Sciences under Award Number DE-SC0001086.

\section{REFERENCES}

[1] Heavens, O., [Optical properties of thin solid films], vol. 2nd edition, Dover Publications Inc. (1992).

[2] Yeh, P., [Optical Waves in Layered Media], Wiley-Blackwell (2005).

[3] Schubert, E. F., [Light-Emitting Diodes], Cambridge University Press (2006).

[4] Kolle, M., Zheng, B., Gibbons, N., Baumberg, J. J., and Steiner, U., "Stretch-tuneable dielectric mirrors and optical microcavities," Optics Express 18(5), 575-581 (2010).

[5] Choi, S. Y., Mamak, M., von Freymann, G., Chopra, N., and Ozin, G. A., "Mesoporous Bragg stack color tunable sensors," Nano Letters 6(11), 2456-2461 (2006).

[6] Fuertes, C., Colodrero, S., Lozano, G., González-Elipe, A. R., Grosso, D., Boissière, C., Sánchez, C., SolerIllia, G. J. d. A., and Míguez, H., "Sorption properties of mesoporous multilayer thin films," Journal of Physical Chemistry C 106, 3157-3163 (2008).

[7] Lin, V., Motesharei, K., Dancil, K., Sailor, M., and Ghadiri, M., "A porous silicon-based optical interferometric biosensor," Science 278(5339), 840-843 (1997).

[8] Bonifacio, L. D., Puzzo, D. P., Breslav, S., Willey, B. M., McGeer, A., and Ozin, G. A., "Towards the photonic nose: a novel platform for molecule and bacteria identification," Advanced Materials 22(12), 13514 (2010). 
[9] Hidalgo, N., Calvo, M. E., and Míguez, H., "Mesostructured thin films as responsive optical coatings of photonic crystals.," Small 5(20), 2309-15 (2009).

[10] Sánchez-Sobrado, O., Calvo, M. E., Núñez, N., Ocaña, M., Lozano, G., and Míguez, H., "Environmentally responsive nanoparticle-based luminescent optical resonators," Nanoscale 2(6), 936 (2010).

[11] Puzzo, D. P., Scotognella, F., Zavelani-Rossi, M., Sebastian, M., Lough, A. J., Manners, I., Lanzani, G., Tubino, R., and Ozin, G. A., "Distributed feedback lasing from a composite poly(phenylene vinylene)nanoparticle one-dimensional photonic crystal.," Nano Letters 9(12), 4273-8 (2009).

[12] Colodrero, S., Mihi, A., Haggman, L., Ocana, M., Boschloo, G., Hagfeldt, A., and Míguez, H., "Porous one-dimensional photonic crystals improve the power-conversion efficiency of dye-sensitized solar cells," Advanced Materials 21(7), 764-770 (2009).

[13] Vincent, G., "Optical-properties of porous silicon superlattices," Applied Physics Letters 64(18), 2367-2369 (1994).

[14] Fuertes, M. C., Lopez-Alcaraz, F. J., Marchi, M. C., Troiani, H. E., Luca, V., Míguez, H., and Arturo Soler-Illia, G. J. d. A., "Photonic crystals from ordered mesoporous thin-film functional building blocks," Advanced Functional Materials 17(8), 1247-1254 (2007).

[15] Wu, Z., Lee, D., Rubner, M. F., and Cohen, R. E., "Structural color in porous, superhydrophilic, and self-cleaning $\mathrm{SiO} 2 / \mathrm{TiO} 2$ Bragg stacks.," Small 3(8), 1445-51 (2007).

[16] Colodrero, S., Ocana, M., and Míguez, H., "Nanoparticle-based one-dimensional photonic crystals," Langmuir 24(9), 4430-4434 (2008).

[17] Calvo, M. E., Colodrero, S., Rojas, T. C., Anta, J. A., Ocana, M., and Míguez, H., "Photoconducting Bragg mirrors based on $\mathrm{TiO}_{2}$ nanoparticle multilayers," Advanced Functional Materials 18, 2708-2715 (2008).

[18] Bonifacio, L. D., Lotsch, B. V., Puzzo, D. P., Scotognella, F., and Ozin, G. A., "Stacking the nanochemistry deck: structural and compositional diversity in one-dimensional photonic crystals," Advanced Materials 21(16), 1641-1646 (2009).

[19] Templin, M., Franck, A., DuChesne, A., Leist, H., Zhang, Y., Ulrich, R., Schadler, V., and Wiesner, U., "Organically modified aluminosilicate mesostructures from block copolymer phases," Science 278(5344), 1795-1798 (1997).

[20] Guldin, S., Kolle, M., Stefik, M., Langford, R., Eder, D., Wiesner, U., and Steiner, U., "Tunable mesoporous bragg reflectors based on block-copolymer self-assembly," Advanced Materials, DOI: 10.1002/adma.201100640 (2011).

[21] Choi, S., Mamak, M., Coombs, N., Chopra, N., and Ozin, G. A., "Thermally stable two-dimensional hexagonal mesoporous nanocrystalline anatase, meso-nc- $\mathrm{TiO}_{2}$ : bulk and crack-free thin film morphologies," Advanced Functional Materials 14(4), 335-344 (2004).

[22] Guldin, S., Docampo, P., Stefik, M., Kamita, G., Wiesner, U., Snaith, H. J., and Steiner, U., "Layer-by-layer formation of block copolymer derived $\mathrm{TiO}_{2}$ for solid state dye-sensitized solar cells," submitted .

[23] Hutchinson, N. J., Coquil, T., Navid, A., and Pilon, L., "Effective optical properties of highly ordered mesoporous thin films," Thin Solid Films 518(8), 2141-2146 (2010).

[24] Guldin, S., Hüttner, S., Kolle, M., Welland, M. E., Müller-Buschbaum, P., Friend, R. H., Steiner, U., and Tétreault, N., "Dye-sensitized solar cell based on a three-dimensional photonic crystal," Nano Letters 10(7), 2303-2309 (2010).

[25] Nedelcu, M., Lee, J., Crossland, E. J. W., Warren, S. C., Orilall, M. C., Guldin, S., Hüttner, S., Ducati, C., Eder, D., Wiesner, U., Steiner, U., and Snaith, H. J., "Block copolymer directed synthesis of mesoporous $\mathrm{TiO}_{2}$ for dye-sensitized solar cells," Soft Matter 5(1), 134-139 (2009).

[26] Nedelcu, M., Guldin, S., Orilall, M. C., Lee, J., Hüttner, S., Crossland, E. J. W., Warren, S. C., Ducati, C., Laity, P. R., Eder, D., Wiesner, U., Steiner, U., and Snaith, H. J., "Monolithic route to efficient dye-sensitized solar cells employing diblock copolymers for mesoporous $\mathrm{TiO}_{2}$," Journal Of Materials Chemistry 20(7), 1261-1268 (2010).

[27] Guldin, S., Hüttner, S., Tiwana, P., Orilall, M. C., Ülgüt, B., Stefik, M., Docampo, P., Kolle, M., Divitini, G., Ducati, C., Redfern, S. A. T., Snaith, H. J., Wiesner, U., Eder, D., and Steiner, U., "Improved conductivity in dye-sensitised solar cells through block-copolymer confined $\mathrm{TiO}_{2}$ crystallisation," Energy $\&$ Environmental Science 4(1), 225-233 (2011). 
[28] Docampo, P., Guldin, S., Stefik, M., Tiwana, P., Orilall, M. C., Hüttner, S., Sai, H., Wiesner, U., Steiner, U., and Snaith, H. J., "Control of solid-state dye-sensitized solar cell performance by block-copolymer-directed $\mathrm{TiO}_{2}$ synthesis," Advanced Functional Materials 20(11), 1787 - 1796 (2010).

[29] Yang, P. and Margolese, D. I., "Generalized syntheses of large-pore mesoporous metal oxides with semicrystalline frameworks," Nature 396, 152-155 (1998).

[30] Sanchez, C., Boissière, C., Grosso, D., Laberty, C., and Nicole, L., "Design, synthesis, and properties of inorganic and hybrid thin films having periodically organized nanoporosity," Chemistry of Materials 20(3), 682-737 (2008). 